\title{
Semi-inner products and operators which attain their norm
}

\author{
By Paweł WóJcik and Tomasz StypuŁA \\ Institute of Mathematics, Pedagogical University of Cracow, Podchorạżych 2, 30-084 Kraków, Poland
}

(Communicated by Kenji FukAYA, M.J.A., Jan. 12, 2021)

\begin{abstract}
It has been proved by Koehler and Rosenthal [Studia Math. 36 (1970), 213$216]$ that an linear isometry $U \in \mathcal{L}(X)$ preserves some semi-inner-product. Recently, similar investigations have been carried out by Niemiec and Wójcik for continuous representations of amenable semigroups into $\mathcal{L}(X)$ (cf. [Studia Math. 252 (2020), 27-48]).

In this paper we generalize the result of Koehler and Rosenthal. Namely, we prove that if an operator $T \in \mathcal{L}(X)$ of norm one attains its norm then there is a semi-inner-product $[\cdot \mid \diamond]: X \times$ $X \rightarrow \mathbf{F}$ that the operator $T$ preserves this semi-inner-product on the norm attaining set. More precisely, we show that the equality $[T(\cdot) \mid T x]=[\cdot \mid x]$ holds for all $x \in M_{T}:=\left\{y \in S_{X}:\|T y\|=1\right\}$.
\end{abstract}

Key words: Semi-inner product; norm attainment; bounded operator.

1. Introduction. Let $(X,\|\cdot\|)$ be a normed space over $\mathbf{F} \in\{\mathbf{R}, \mathbf{C}\}$. Lumer [5] and Giles [3] proved that in a normed space $X$ there always exists a mapping $[\cdot \mid \diamond]: X \times X \rightarrow \mathbf{F}$ satisfying the following properties:

(sip1) $\forall_{x, y, z \in X} \forall_{\alpha \in \mathbf{F}}:[\alpha x+y \mid z]=\alpha[x \mid z]+[y \mid z] ;$

(sip2) $\forall_{x, y \in X} \forall_{\alpha \in \mathbf{F}}: \quad[x \mid \alpha y]=\bar{\alpha}[x \mid y]$;

(sip3) $\forall_{x, y \in X}: \quad|[x \mid y]| \leqslant\|x\| \cdot\|y\|$;

$(\operatorname{sip} 4) \forall_{x \in X}: \quad[x \mid x]=\|x\|^{2}$.

Such a mapping is called a semi-inner-product in $X$ (generating the norm $\|\cdot\|$ ). There may exist infinitely many different semi-inner-products in $(X,\|\cdot\|)$. Indeed, non-smooth spaces furnish the necessary examples. However, the above four conditions say that semi-inner-products are perfect generalizations of inner products.

Let $\mathcal{L}(X)$ be the space of all linear and bounded operators on a normed space $X$. Koehler and Rosenthal [4] gave the following characterization of linear isometries in $\mathcal{L}(X)$.

Theorem 1.1 [4]. Let $X$ be a normed space (real or complex) and let $U \in \mathcal{L}(X)$ be an isometry. Then there is a semi-inner-product $[\cdot \mid \diamond]_{U}$ such that

$$
\forall x, y \in X \quad[U y \mid U x]_{U}=[y \mid x]_{U} .
$$

Recently, Niemiec and Wójcik [6, Theorem 4.13.] provided some extension of Theorem 1.1. Their result dealt with continuous representations

2010 Mathematics Subject Classification. Primary 46B20, 46C50; Secondary 46B10, 47A05. of amenable semigroups into the algebra $\mathcal{L}(X)$ (see [6] for the definition of amenable semigroups and amenability).

The main aim of this paper is to provide another extension of the result of Koehler and Rosenthal. Namely, we will consider an operator $T$ which attains its norm instead of an isometry $U$. Then we will prove that a similar version of (1) holds on the norm attainment set of $T$.

This will not be only shallow change. We show the proof for general operator which is more difficult than in the case of isometries.

Moreover, it is worth mentioning that norm attainment sets has been widely investigated. Pioneering results, as well as more information on normed attaining sets, can be found, e.g. in [7].

Let us fix our main notations and terminologies. The set $\{0,1,2,3, \ldots\}$ will be denoted by $\mathbf{N}_{0}$. $X^{*}$ denotes the dual space of $X . S_{X}$ stands for the unit sphere in $X$. For $x \in X$ let $J(x)$ denote the set of its supporting functionals:

$$
J(x):=\left\{f \in X^{*}:\|f\|=1, f(x)=\|x\|\right\} .
$$

The Hahn-Banach Theorem implies that $J(x) \neq \emptyset$ for all $x \in X \backslash\{0\}$.

For a given $T \in \mathcal{L}(X)$, we denote

$$
M_{T}:=\left\{x \in S_{X}:\|T x\|=\|T\|\right\},
$$

i.e. the set of points on the unit sphere at which $T$ attains its norm. Generally, $M_{T}$ may be empty; however, if $X$ is a reflexive Banach space, then compactness of $T$ implies $M_{T} \neq \emptyset$. 
Moreover, we have also the following constant

$$
\begin{aligned}
\gamma(T) & :=\inf \left\{\|T x\|: x \in S_{X}\right\} \\
& =\sup \left\{c \geq 0: \forall_{x \in X} c\|x\| \leq\|T x\|\right\},
\end{aligned}
$$

and another subset of the unit sphere

$$
m_{T}:=\left\{x \in S_{X}:\|T x\|=\gamma(T)\right\} .
$$

It is easy to see that the inequality $\gamma(T)>0$ implies the injectivity of $T$.

2. Extension of the Koehler-Rosenthal theorem. In this section we will prove a sharpened version of the Koehler-Rosenthal result. We start with an auxiliary result.

Theorem 2.1. Suppose that $z \in m_{T}, \gamma(T)>$ 0 and $g \in J(z)$. Then there is a functional $h_{z} \in J(T z)$ such that $\gamma(T) g=h_{z} \circ T$.

Proof. It is easy to check that $\operatorname{span}\{z\} \cap$ ker $g=\{0\}$. Since $\gamma(T)>0, T$ is injective. So, we get $\operatorname{span}\{T z\} \cap T(\operatorname{ker} g)=\{0\}$.

Now, we can define a linear functional $h$ : $\operatorname{span}\{T z\}+T(\operatorname{ker} g) \rightarrow \mathbf{F} \quad$ by $\quad h(\alpha T z+T k):=$ $\alpha \gamma(T)$, where $\alpha \in \mathbf{F}$ and $k \in \operatorname{ker} g$. Then we have

$$
\begin{aligned}
|h(\alpha T z+T k)| & =|\alpha| \gamma(T)=|\alpha| \gamma(T)\|z\| \\
& =|\alpha| \gamma(T) g(z)=\gamma(T)|g(\alpha z)| \\
& =\gamma(T)|g(\alpha z+k)| \\
& \leq \gamma(T)\|\alpha z+k\| \stackrel{(2)}{\leq}\|\alpha T z+T k\| .
\end{aligned}
$$

Thus $\|h\| \leq 1$. On the other hand $\left|h\left(\frac{T z}{\|T z\|}\right)\right|=$ $\frac{1}{\|T z\|} \gamma(T)=1$, therefore $\|h\|=1$. Now the HahnBanach Theorem implies that there exists a linear functional $h_{z}: X \rightarrow \mathbf{F}$ such that $\left\|h_{z}\right\|=1$ and $\left.h_{z}\right|_{\operatorname{span}\{T z\}+T(\operatorname{ker} g)}=h$. From this we obtain $h_{z}(T z)=$ $h(T z)=\gamma(T)=\|T z\|$ thus $h_{z} \in J(T z)$. It is easily seen that $T(\operatorname{ker} g) \subseteq \operatorname{ker} h_{z}$. It follows from this inclusion that $\operatorname{ker} g \subseteq \operatorname{ker}\left(h_{z} \circ T\right)$. But $h_{z} \circ T \neq 0$. This is proved by writing $\left(h_{z} \circ T\right)(z)=h_{z}(T z)=$ $\gamma(T)>0$.

Observe that codim ker $g=1=\operatorname{codim} \operatorname{ker}\left(h_{z} \circ\right.$ $T)$. Therefore we obtain $\operatorname{ker} g=\operatorname{ker}\left(h_{z} \circ T\right)$. This implies $\alpha g=h_{z} \circ T$ with some $\alpha \in \mathbf{F}$. From this equality we deduce $\alpha=\alpha g(z)=h_{z}(T z)=\gamma(T)$.

We are in position to prove an extension of the Koehler-Rosenthal theorem.

Theorem 2.2. Suppose that $T \in \mathcal{L}(X)$ is an operator such that $\|T\|=1$ and $M_{T} \neq \emptyset$. Then there is a semi-inner-product $[\cdot \mid \diamond]_{T}: X \times X \rightarrow \mathbf{F}$ such that

$$
\forall_{x \in M_{T}} \forall_{y \in X} \quad[T y \mid T x]_{T}=[y \mid x]_{T} .
$$

Moreover, if $T$ is not an isometry and $m_{T} \neq \emptyset$ and $\gamma(T)>0$, then there exists a semi-inner-product $[\cdot \mid \diamond]_{T}: X \times X \rightarrow \mathbf{F}$ such that

$$
\begin{aligned}
\forall_{x \in M_{T}} \forall_{u \in m_{T}} \forall_{y \in X}[T y \mid T x]_{T} & =[y \mid x]_{T} \text { and } \\
{[T y \mid T u]_{T} } & =\gamma(T)^{2}[y \mid u]_{T} .
\end{aligned}
$$

Proof. Since the proofs are similar we present only the second part of theorem. The first one is easier. So, assume that $M_{T} \neq m_{T}$ and $\gamma(T)>0$. The proof is long, therefore we find it convenient to write the six steps of the proof.

Step I. First, we define a subset

$$
A:=\left\{x \in M_{T}: T x \notin M_{T}\right\} \subseteq M_{T} .
$$

Next, fix $x \in A$ and let us consider a subset

$$
D_{x}:=\left\{y \in M_{T}: \exists_{n \in \mathbf{N}_{0}} T^{n} y=x\right\} \subseteq M_{T} .
$$

We see at once that $A \subseteq D_{x}$ and $T\left(D_{x}\right) \subseteq D_{x} \cup$ $T(A)$. It is easy to check that

$$
\begin{aligned}
& \text { if } z \in M_{T} \text { and } T^{n} z \in M_{T}, \\
& \text { then } T^{k} z \in M_{T} \text { for all } k \leq n .
\end{aligned}
$$

Therefore, one should be able to verify with little effort that

(5) if $x_{1}, x_{2} \in A$ and $x_{1} \neq x_{2}$, then $D_{x_{1}} \cap D_{x_{2}}=\emptyset$.

The property (5) will be important later. Now we define the next two subsets

$$
\begin{aligned}
& K:=\left\{y \in M_{T}: \forall_{n \in \mathbf{N}_{0}} T^{n} y \in M_{T}\right\} \text { and } \\
& \widehat{K}:=\left\{y \in M_{T}: \exists_{n \in \mathbf{N}_{0}} T^{n} y \notin M_{T}\right\} .
\end{aligned}
$$

A moment's reflection shows that

$$
M_{T}=K \cup \widehat{K}=K \cup \bigcup_{x \in A} D_{x} .
$$

For fixed $x \in A$ and $y \in D_{x} \cup T(A)$, we define a constant

$$
p(y, x):=\min \left\{n \in \mathbf{N}_{0}: T^{n} y=T x\right\} .
$$

In particular, $p(x, x)=1$ and $p(T x, x)=0$ and $T^{p(y, x)} y=T x$. Moreover, if $T y \in D_{x} \cup T(A)$, then we obtain

(6) $p(T y, x)=\min \left\{n \in \mathbf{N}_{0}: T^{n}(T y)=T x\right\}$

$$
\begin{aligned}
& =\min \left\{n \in \mathbf{N}_{0}: T^{n+1} y=T x\right\} \\
& =\min \left\{k \in \mathbf{N}_{0}: T^{k} y=T x\right\} \\
& =-1+\min \left\{k \in \mathbf{N}_{0}: T^{k} y=T x\right\} \\
& =-1+p(y, x) .
\end{aligned}
$$

For each element $x \in A$, using the axiom of choice, we take an arbitrary $f_{T x} \in J(T x)$ such that 
(7) if $x, x^{\prime} \in A, J(T x)=J\left(T x^{\prime}\right)$, then $f_{T x}=f_{T x^{\prime}}$.

The above remark will play a significant role in this proof. Since $\|T x\|=1$, we see that $f_{T x}(T x)=1$. If $y \in D_{x}$ and $T y \in D_{x} \cup T(A)$, then

(8) $f_{T x}\left(T^{p(T y, x)+1} y\right)=f_{T x}\left(T^{p(y, x)} y\right)=f_{T x}(T x)=1$.

It is obvious that $\left\|f_{T x} \circ T^{p(T y, x)+1}\right\| \leq 1$. So, applying (8) we obtain

$$
\left\|f_{T x} \circ T^{p(T y, x)+1}\right\|=1 .
$$

Step II. Before defining a semi-inner-product $[\cdot \mid \diamond]_{T}$, it is convenient to introduce a mapping $[\cdot \mid \diamond]_{S}$ : $X \times S_{X} \rightarrow \mathbf{F}$. First we define it on

$$
X \times\left(\bigcup_{x \in A} D_{x} \cup T(A)\right) .
$$

So let $y \in \bigcup_{x \in A} D_{x} \cup T(A)$. It is convenient and, more importantly, helpful to recall that $D_{x} \cap T(A)=\emptyset$.

If $y \in \bigcup_{x \in A} D_{x}$, then we conclude from (5) that there is a unique $x \in A$ such that $y \in D_{x}$. On the other hand, if $y \in T(A)$, there is a unique (by the injectivity of $T$ ) vector $x \in A$ such that $y=T x$.

These remarks allow us to introduce the following definition

$$
[w \mid y]_{S}:=\left(f_{T x} \circ T^{p(y, x)}\right)(w) \text { for } w \in X .
$$

Before returning into the proof, a few words are appropriate. More precisely: if $\gamma(T)>0$ were not assumed, we would prove only (3). But then $T$ need not be injective. Therefore it is necessary to check that $[\cdot \mid \diamond]_{\mathrm{S}}$ is well defined on $T(A)$. To do this we assume that $x, x^{\prime} \in A$ and $y=T x=T x^{\prime}$. Then

$$
\begin{aligned}
& {[w \mid T x]_{S} \stackrel{(10)}{=}\left(f_{T x} \circ T^{p(T x, x)}\right)(w)=\left(f_{T x} \circ T^{0}\right)(w)} \\
& \stackrel{(7)}{=}\left(f_{T x^{\prime}} \circ T^{p\left(T x^{\prime}, x^{\prime}\right)}\right)(w) \stackrel{(10)}{=}\left[w \mid T x^{\prime}\right]_{S} .
\end{aligned}
$$

Thus $[\cdot \mid \diamond]_{S}$ is well defined on $T(A)$ in each case. To end this step, we fix a vector $y \in D_{x}$ such that $T y \in$ $D_{x} \cup T(A)$ and we write

$$
\begin{aligned}
{[w \mid y]_{S} } & \stackrel{(10)}{=} f_{T x}\left(T^{p(y, x)} w\right) \stackrel{(6)}{=} f_{T x}\left(T^{p(T y, x)+1} w\right) \\
& =f_{T x}\left(T^{p(T y, x)}(T w)\right) \stackrel{(10)}{=}[T w \mid T y]_{S} .
\end{aligned}
$$

Step III. From now on, the set $m_{T}$ plays a main role. Since $T$ is not an isometry, $M_{T} \neq m_{T}$. It is easy to see that $\frac{1}{\gamma(T)} T\left(m_{T}\right) \subseteq S_{X}$. Now we define a mapping $[\cdot \mid \diamond]_{S}$ on the set $X \times\left(m_{T} \cup \frac{1}{\gamma(T)} T\left(m_{T}\right)\right)$. Note that

$$
\begin{aligned}
& m_{T}=\left(m_{T} \cap T\left(M_{T}\right)\right) \cup\left(m_{T} \backslash T\left(M_{T}\right)\right) \quad \text { and } \\
& \emptyset=\left(m_{T} \cap T\left(M_{T}\right)\right) \cap\left(m_{T} \backslash T\left(M_{T}\right)\right) .
\end{aligned}
$$

First we consider the subset $m_{T} \cap T\left(M_{T}\right)$. Fix $x \in$ $M_{T}$ such that $T x \in m_{T}$, i.e. $T x \in m_{T} \cap T\left(M_{T}\right)$. Then $x \in A$ and the functional $f_{T x} \in J(T x)$ is defined as above.

So, for each element $x \in A$, using the axiom of choice and Theorem 2.1, we take an arbitrary $h_{T x} \in$ $J\left(T^{2} x\right)$ such that

(13) if $x, x^{\prime} \in A$ and $T x=T x^{\prime}$, then $h_{T x}=h_{T x^{\prime}}$,

and

$$
\gamma(T) f_{T x}=h_{T x} \circ T .
$$

Now fix $w \in X$. Since $T x \in m_{T}, \frac{T(T x)}{\|T(T x)\|} \in S_{X}$. Let us consider the next definition:

$$
\left[w \mid \frac{T(T x)}{\|T(T x)\|}\right]_{S}:=h_{T x}(w) \quad \text { for } w \in X .
$$

It follows from (13) that the equality (15) is well defined. Furthermore: since $T x \in m_{T}, T x \notin M_{T}$. However, we know that $x \in M_{T}$. Thus we conclude that $x \in D_{x}$. Therefore, we obtain

$$
\begin{aligned}
{\left[T w \mid \frac{T(T x)}{\|T(T x)\|}\right]_{S} } & \stackrel{(15)}{=} h_{T x}(T w) \stackrel{(14)}{=} \gamma(T) f_{T x}(w) \\
& =\gamma(T) f_{T x}\left(T^{0} w\right) \\
& =\gamma(T) f_{T x}\left(T^{p(T x, x)} w\right) \\
& \stackrel{(10)}{=} \gamma(T)[w \mid T x] .
\end{aligned}
$$

Now we investigate the subset $m_{T} \backslash T\left(M_{T}\right)$. So, for each $x \in m_{T} \backslash T\left(M_{T}\right)$, using, again, the axiom of choice, we take an arbitrary $g_{x} \in J(x)$. Now Theorem 2.1 and the axiom of choice imply that there is a functional $h_{x} \in J(T x)$ such that

$$
\gamma(T) g_{x}=h_{x} \circ T \quad \text { (in particular, }\left\|h_{x} \circ T\right\|=\gamma(T) \text { ) }
$$

and if $x, x^{\prime} \in A$ and $T x=T x^{\prime}$, then $h_{x}=h_{x^{\prime}}$.

Now fix $w \in X$. Clearly, $\frac{T x}{\|T x\|} \in S_{X}$. Then we introduce another definition:

$$
\begin{aligned}
& {[w \mid x]_{S}:=\frac{1}{\gamma(T)} h_{x}(T w) \text { and }} \\
& {\left[w \mid \frac{T x}{\|T x\|}\right]_{S}:=h_{x}(w) .}
\end{aligned}
$$

In the same manner as before we can see that the second part of (17) is well defined. So, we get 


$$
\left[T w \mid \frac{T x}{\|T x\|}\right]_{S} \stackrel{(17)}{=} h_{x}(T w) \stackrel{(17)}{=} \gamma(T)[w \mid x]_{S} .
$$

Since $\left\|h_{x} \circ T\right\|=\gamma(T)$, (17) implies $[\cdot \mid x]_{S} \in X^{*}$ and $\left|[\cdot \mid x]_{S}\right| \leq\|\cdot\|$ and $[x \mid x]_{S}=\frac{1}{\gamma(T)} h_{x}(T x)=g_{x}(x)=1$.

Step $I V$. The above parts will be summarized. Define a subset $\mathfrak{M} \subseteq S_{X}$ by the formula

$$
\mathfrak{M}:=\widehat{K} \cup m_{T} \cup T(\widehat{K}) \cup \frac{1}{\gamma(T)} T\left(m_{T}\right) .
$$

Combining the end of Step III, (9), (10) and (15), we immediately get

$$
[\cdot \mid x]_{S} \in X^{*} \text { and }\left|[\cdot \mid x]_{S}\right| \leq\|\cdot\| \quad \text { for } x \in \mathfrak{M},
$$

and moreover

$$
[x \mid x]_{S}=1 \quad \text { for all } x \in \mathfrak{M} .
$$

So, we may consider the mapping $[\cdot \mid \diamond]_{S}: X \times \mathfrak{M} \rightarrow \mathbf{F}$ as well defined.

Step $V$. Now fix a semi-inner-product $[\cdot \mid \diamond]_{0}$ : $X \times X \rightarrow \mathbf{F}$. A trivial verification shows that $\left(\left[T^{n} b \mid\right.\right.$ $\left.\left.T^{n} a\right]_{o}: n \in\{0,1,2, \ldots\}\right)$ is a bounded sequence for each fixed $a, b \in X$. Fix a Banach limit $L: l^{\infty} \rightarrow \mathbf{F}$.

Now, we are able to extend the mapping $[\cdot \mid \diamond]_{S}: X \times \mathfrak{M} \rightarrow \mathbf{F}$ to the set $X \times S_{X}$ by the formula

$$
\begin{aligned}
& {[w \mid a]_{S}} \\
& :=\left\{\begin{array}{l}
{[w \mid a]_{S} \text { if } a \in \mathfrak{M},} \\
L\left([w \mid a]_{o},[T w \mid T a]_{o},\left[T^{2} w \mid T^{2} a\right]_{o}, \ldots\right) \\
\text { if } a \in K, \\
{[w \mid a]_{o} \text { if } a \in S_{X} \backslash(\mathfrak{M} \cup K) .}
\end{array}\right.
\end{aligned}
$$

If $a \in K$, then the shift property of the Banach limit shows that

$$
\begin{aligned}
{[w \mid a]_{S} } & =L\left(\left[T^{n} w \mid T^{n} a\right]_{o}: n \in\{0,1,2, \ldots\}\right) \\
& =L\left(\left[T^{n+1} w \mid T^{n+1} a\right]_{o}: n \in\{0,1,2, \ldots\}\right) \\
& =[T w \mid T a]_{S} .
\end{aligned}
$$

Step VI. Finally, we extend the mapping $[\cdot \mid \diamond]_{S}$ to $[\cdot \mid \diamond]_{T}: X \times X \rightarrow \mathbf{F}$ by the formulas

$$
[b \mid a]_{T}:=\|a\| \cdot\left[b \mid \frac{a}{\|a\|}\right]_{S} \text { and }
$$$$
\text { we put }[b \mid a]_{T}:=0 \text { if } a=0 .
$$

It is easy to see that $[\cdot \mid \diamond]_{T}$ satisfies (sip2). It follows from (19) and (21) that $[\cdot \mid \diamond]_{T}$ satisfies (sip1) and (sip3).

Furthermore, the conditions (20) and (21) say that $[\cdot \mid \diamond]_{T}$ satisfies (sip4), thus it is a semiinner-product.
Combining (11), (16), (18) and (22), we immediately get (4).

To end this section we present some application of Theorem 2.2. The next two theorems concern the Birkhoff orthogonality and the semiorthogonality.

Theorem 2.3 ([2, Theorem 57, p. 140]). Let $(X,\|\cdot\|)$ be a normed space, $E$ its linear subspace and $x_{o} \in X \backslash E$. Then the following statements are equivalent:

(a) $x \perp_{\mathrm{B}} E$, i.e. $\forall_{e \in E}\left\|x_{o}\right\| \leq\left\|x_{o}+e\right\|$;

(b) There exists a semi-inner-product $[\cdot \mid \bullet]$ which generates the norm $\|\cdot\|$ and for which $x_{o} \perp_{\mathrm{s}} E$, i.e. $\forall_{e \in E}\left[e \mid x_{o}\right]=0$. follows:

The above theorem can be strengthen as

Theorem 2.4. Let $(X,\|\cdot\|)$ be a normed space. Let $C, E \subseteq X$ be nontrivial closed linear subspaces such that $X=C+E$. Then the following statements are equivalent:

(a) $C \perp_{\mathrm{B}} E$, i.e. $\forall_{c \in C} \forall_{e \in E}\|c\| \leq\|c+e\|$;

(b) There exists a semi-inner-product $[\cdot \mid \diamond]$ which generates the norm $\|\cdot\|$ and for which $C \perp_{\mathrm{s}} E$, i.e. $\forall_{c \in C} \forall_{e \in E}[e \mid c]=0$;

Proof. Assuming (a) we obtain $C \cap E=\{0\}$. For each vector $c+e \in C+E=X$ (unique decomposition) we define $P(c+e):=c$. It is obvious that $P$ is linear. Applying (a) yields

$$
\|P(c+e)\|=\|c\| \leq\|c+e\| .
$$

Thus we get $\|P\| \leq 1$. But $\left\|P\left(\frac{c}{\|c\|}\right)\right\|=\left\|\frac{c}{\|c\|}\right\|=1$. Hence $\|P\|=1$.

Theorem 2.2 now shows that there exists a semi-inner-product $[\cdot \mid \diamond]_{P}$ such that $[P(\cdot) \mid P x]_{P}=$ $[\cdot \mid x]_{P}$ for all $x \in M_{P}$. We check at once that $M_{P} \supseteq$ $S_{X} \cap C$. It means that, for any $c \in C \backslash\{0\}$ and $e \in E, \quad$ we have $0=\left[0 \mid P\left(\frac{c}{\|c\|}\right)\right]_{P}=\left[P e \mid P\left(\frac{c}{\| c \mid}\right)\right]_{P}=$ $\left[e \mid \frac{c}{\| c \mid}\right]_{P}=\frac{1}{\|c\|}[e \mid c]$. Hence $0=[e \mid c]$. So the implication (a) $\Rightarrow(\mathrm{b})$ is proved. The reverse is true, since $\perp_{\mathrm{s}} \subseteq \perp_{\mathrm{B}}$ (see e.g. [2]).

3. Norm derivatives on norm attaintment set. From now on, we assume that the considered normed spaces are real. This section is in spirit to Theorem 2.2. Namely, instead of semi-inner-product, we consider norm derivatives, which are defined by the formulas $\rho_{+}^{\prime}(x, y):=$ $\lim _{t \rightarrow 0^{+}} \frac{\|x+t y\|^{2}-\|x\|^{2}}{2 t}$ and $\rho_{-}^{\prime}(x, y):=\lim _{t \rightarrow 0^{-}} \frac{\|x+t y\|^{2}-\|x\|^{2}}{2 t}$ (cf. $[2,1]$ for the background and properties of these functionals). In particular, we have 


$$
\text { (nd1) } \begin{aligned}
\forall_{x, y} & \forall_{\alpha \in \mathbf{R}}: \rho_{+}^{\prime}(x, \alpha x+y) \\
= & \alpha \rho_{+}^{\prime}(x, x)+\rho_{+}^{\prime}(x, y) ;
\end{aligned}
$$

(nd2) $\forall_{x, y} \forall_{\alpha \geq \mathbf{R}}: \rho_{ \pm}^{\prime}(x, \alpha y)=\alpha \rho_{ \pm}^{\prime}(x, y)=\rho_{ \pm}^{\prime}(\alpha x, y)$;

(nd3) $\forall_{x, y} \forall_{\alpha<\mathbf{R}}: \rho_{ \pm}^{\prime}(x, \alpha y)=\alpha \rho_{\mp}^{\prime}(x, y)=\rho_{ \pm}^{\prime}(\alpha x, y)$;

(nd4) $\forall_{x, y}:\left|\rho_{ \pm}^{\prime}(x, y)\right| \leqslant\|x\| \cdot\|y\|, \quad \rho_{ \pm}^{\prime}(x, x)=\|x\|^{2}$;

(nd5) $\forall_{x, y}: \rho_{-}^{\prime}(x, y) \leq \rho_{+}^{\prime}(x, y)$.

Let us recall a useful connection between norm derivatives and the semi-inner-products (cf. [2, Theorem 15, p. 36]). Let $\mathcal{S I P}_{X}$ denote the set of all semi-inner-products in the normed space $(X,\|\cdot\|)$ which generate the norm $\|\cdot\|$. Then

$$
\begin{aligned}
\rho_{+}^{\prime}(x, y) & =\|x\| \cdot \sup \{\varphi(y): \varphi \in J(x)\} \\
& =\sup \left\{[y \mid x]:[\cdot \mid \diamond] \in \operatorname{SIP}_{X}\right\} .
\end{aligned}
$$

Note that, if $x \in S_{X}$ then $\rho_{-}^{\prime}(x, y) \leqslant \varphi(y) \leqslant \rho_{+}^{\prime}(x, y)$, for every $\varphi \in J(x)$. It is convenient and, more importantly, helpful to prove the following lemma.

Lemma 3.1. Suppose that $X$ is a real normed space. Let $x, y \in X$. Then

$$
\begin{aligned}
\rho_{+}^{\prime}(x, y) & =\|x\| \cdot \max \{\varphi(y): \varphi \in J(x)\} \\
& =\max \left\{[y \mid x]:[\cdot \mid \diamond] \in \mathcal{S I P}_{X}\right\}, \\
\rho_{-}^{\prime}(x, y) & =\|x\| \cdot \min \{\varphi(y): \varphi \in J(x)\} \\
& =\min \left\{[y \mid x]:[\cdot \mid \diamond] \in \mathcal{S I P}_{X}\right\} .
\end{aligned}
$$

Proof. It is easy to check that the function $J(x) \ni \varphi \mapsto \varphi(y) \in \mathbf{R}$ is weak $^{*}$-continuous. Moreover, the set $J(x)$ is weak*-compact. Therefore the function $J(x) \ni \varphi \mapsto \varphi(y) \in \mathbf{R}$ attains its maximum and minimum. So, the first equality is proved. Since $\rho_{+}^{\prime}(x,-y)=-\rho_{-}^{\prime}(x, y)$, we get the second one.

Theorem 3.2. Let $T \in \mathcal{L}(X, Y)$ be a linear operator between two real normed spaces. Suppose that $\|T\|=1$. Then the following inequalities are true:

$$
\begin{aligned}
& \forall_{x \in M_{T}} \forall_{y \in X} \\
& \rho_{-}^{\prime}(x, y) \leqslant \rho_{-}^{\prime}(T x, T y) \leqslant \rho_{+}^{\prime}(T x, T y) \leqslant \rho_{+}^{\prime}(x, y) .
\end{aligned}
$$

Proof. By (nd5) the middle inequality holds true in general, so it remains to prove the outer ones. Fix $x \in M_{T}$ and $y \in X$. Choose arbitrarily $f \in J(T x)$. By the definition of the set $J(T x)$, we have that $1=(f \circ T)(x) \leqslant\|f \circ T\| \leqslant\|f\| \cdot\|T\|=1$. Therefore $\|f \circ T\|=1$. Thus $f \circ T \in J(x)$. So we conclude from Lemma 3.1 that we have

$$
\rho_{-}^{\prime}(x, y) \leqslant(f \circ T)(y) \leqslant \rho_{+}^{\prime}(x, y) .
$$

This proves that the inequalities

$$
\rho_{-}^{\prime}(x, y) \leqslant f(T y) \leqslant \rho_{+}^{\prime}(x, y)
$$

are true for all $f \in J(T x)$. Passing to the infimum and to the supremum over $J(T x)$, and using Lemma 3.1 , we get $\rho_{-}^{\prime}(x, y) \leqslant \rho_{-}^{\prime}(T x, T y)$ and $\rho_{+}^{\prime}(T x, T y) \leqslant$ $\rho_{+}^{\prime}(x, y)$, respectively. The proof is complete.

Theorem 3.3. Let $T \in \mathcal{L}(X, Y)$ be a linear operator with $\|T\|=1$ and $m_{T} \neq \emptyset$. Then the following inequalities are true:

$$
\begin{gathered}
\forall_{x \in m_{T}} \forall_{y \in X} \rho_{-}^{\prime}(T x, T y) \leqslant \gamma(T)^{2} \rho_{-}^{\prime}(x, y) \text { and } \\
\gamma(T)^{2} \rho_{+}^{\prime}(x, y) \leqslant \rho_{+}^{\prime}(T x, T y) .
\end{gathered}
$$

Proof. If $\gamma(T)=0$, then $T x=0$ for all $x$ in $m_{T}$ and $\rho_{ \pm}^{\prime}(T x, T(\cdot))=0$. Assume now that $\gamma(T)>0$, and think of $T$ as a mapping from $X$ to $T(X)$. From this point of view, $T \in \mathcal{L}(X, T(X))$ is invertible and it follows that $\gamma(T) T^{-1} \in \mathcal{L}(T(X), X)$ and $\left\|\gamma(T) T^{-1}\right\|=1$. It is not hard to show

$$
\frac{1}{\gamma(T)} T\left(m_{T}\right) \subseteq M_{\gamma(T) T^{-1}}
$$

Applying Theorem 3.2 we get that

$$
\begin{aligned}
\rho_{-}^{\prime}(u, w) & \leqslant \rho_{-}^{\prime}\left(\gamma(T) T^{-1} u, \gamma(T) T^{-1} w\right) \\
& \leqslant \rho_{+}^{\prime}\left(\gamma(T) T^{-1} u, \gamma(T) T^{-1} w\right) \leqslant \rho_{+}^{\prime}(u, w)
\end{aligned}
$$

for all $u \in M_{\gamma(T) T^{-1}}$ and $w \in T(X)$; and we may consider Theorem 3.3 as shown.

\section{References}

[ 1 ] C. Alsina, J. Sikorska and M. S. Tomás, Norm derivatives and characterizations of inner product spaces, World Scientific Publishing Co. Pte. Ltd., Hackensack, NJ, 2010.

[ 2 ] S. S. Dragomir, Semi-inner products and applications, Nova Science Publishers, Inc., Hauppauge, NY, 2004.

[ 3 ] J. R. Giles, Classes of semi-inner-product spaces, Trans. Amer. Math. Soc. 129 (1967), 436-446.

[ 4 ] D. Koehler and P. Rosenthal, On isometries of normed linear spaces, Studia Math. 36 (1970), 213-216.

[ 5 ] G. Lumer, Semi-inner-product spaces, Trans. Amer. Math. Soc. 100 (1961), 29-43.

[6] P. Niemiec and P. Wójcik, Applications of amenable semigroups in operator theory, Studia Math. 252 (2020), no. 1, 27-48.

[ 7 ] D. Sain, On the norm attainment set of a bounded linear operator, J. Math. Anal. Appl. 457 (2018), no. 1, 67-76. 